\title{
THE PREPARATION OF TYPESCRIPTS
}

The attention of authors is particularly directed to the following requests:

\section{Typescript}

Papers should be typed, double-spaced, on one side of white paper (of which A4, 210 by $297 \mathrm{~mm}$, is a suitable size). The pages must be numbered. Generous margins should be left at the side, top and bottom of each page. The copy sent must be clear.

A cover page should give the title, the author's name and institution, with the address to which mail is to be sent.

The title, while brief, must be informative (e.g. A new proof of the ergodic theorem, whereas Some applications of a theorem of Birkhoff would be useless).

The first paragraph or two should form a summary of the main theme of the paper, providing an abstract intelligible to mathematicians.

For a typescript to be accepted for publication, it must accord with the standard requirements of publishers, and be presented in a form in which the author's intentions regarding symbols etc. are clear to a printer (who is not a mathematician).

The following notes are intended to help the author in preparing the typescript. New authors may well enlist the help of senior colleagues, both as to the substance of their work and the details of setting it out correctly and attractively.

\section{Notation}

Notation should be chosen carefully so that mathematical operations are expressed with all possible neatness, to lighten the task of the compositor and reduce the chance of error.

For instance $n_{k}$ ( $n$ sub $k$ ) is common usage, but avoid if possible using $c$ sub $n$ sub $k$. Fractions are generally best expressed by a solidus. Complicated exponentials like

$$
\exp \left\{z^{2} \sin \theta /\left(1+y^{2}\right)\right\}
$$

should be shown in this and no other way.

In the typescript, italics, small capitals and capitals are specified by single, double and triple underlining. Bold-faced type is shown by wavy underlining; wavy will be printed wavy.

It helps if displayed equations or statements which will be quoted later are numbered in order on the right of their line. They can then be referred to by, for example, 'from (7)'.

The author must enable the printer (if necessary by pencilled notes in the margin) to distinguish between similar symbols such as $o, O, 0,0,0 ; x$, $\mathrm{X}, \mathrm{X} ; \phi, \Phi, \varnothing ; \mathrm{I}, 1 ; \varepsilon, \in ; \kappa, k$.

Greek letters can be denoted by $\mathrm{Gk}$ in the margin.
If an author wishes to mark the end of the proof of a theorem, the sign $\square$ may be used.

Footnotes should be avoided.

\section{Diagrams}

It is extremely helpful if diagrams are drawn in Indian ink on white card, faintly blue or greenlined graph paper, or tracing cloth or paper. Symbols, legends and captions should be given on a transparent overlay. Each text figure must be numbered as Figure 1 , Figure $2, \ldots$ and its intended position clearly indicated in the typescript:

Figure 1 here

The author's name in pencil must be on all separate sheets of diagrams.

A figure is expensive to reproduce and should be included only when the subject matter demands it, or when it greatly clarifies the exposition.

The publisher recognizes that some authors do not have the facilities for producing drawings of a sufficiently high standard to be reproduced directly and is therefore willing to have such diagrams re-drawn, provided that they are clear.

\section{Tables}

Tables should be numbered (above the table) and set out on separate sheets. Indicate the position of each in the text as for figures:

Table 3 here

\section{References}

References should be collected at the end of the paper numbered in alphabetical order of the authors' names. A reference to a book should give the title, in italics, and then in roman type the publisher's name and the place and year of publication:

[4] N. Dunford \& J. T. Schwartz Linear Operators Part I. Wiley: New York, 1958.

A reference to a paper should give in italics the title of the periodical, the number of the volume and year, and the beginning and end pages of the paper. Titles should be abbreviated as in Mathematical Reviews:

[6] J. E. Littlewood. The 'pits effect' for functions in the unit circle. J. Analyse Math. 23 (1970), 236-268. 


\title{
Ergodic theory and dynamical systems
}

\author{
VOLUME 3 PART 2 JUNE 1983
}

\section{CONTENTS}

Abels, $H$. Which groups act distally?

Bratteli, O. and Evans, D. E. Dynamical semigroups commuting with compact abelian actions

Guillemin, V. and Sternberg, S. On collective complete integrability according to the method of Thimm

Hurley, $M$. Bifurcation and chain recurrence

Levitt, $G$. Flots topologiquement transitifs sur les surfaces compactes sans bord: contrexemples à une conjecture de Katok

McCluskey, $\mathrm{H}$. and Manning, A. Hausdorff dimension for horseshoes

Pugh, C. C. and Robinson, C. The $C^{1}$ Closing Lemma, including Hamiltonians

Smillie, J. Periodic points of surface homeomorphisms with zero entropy

(C) Cambridge University Press 1983

CAMBRIDGE UNIVERSITY PRESS

The Pitt Building, Trumpington Street, Cambridge CB2 1RP

32 East 57th Street, New York, NY 10022

Printed in Great Britain by J. W. Arrowsmith Ltd, Bristol 